\section{Clinical Practice Guidelines Published for Treatment of Drug-Susceptible Tuberculosis}

The American Thoracic Society, CDC, and the Infectious Diseases Society of America (IDSA) have jointly sponsored the development of guidelines for the treatment of drugsusceptible tuberculosis, which were published by IDSA in Clinical Infectious Diseases on August 11, 2016 (1) and are available through IDSA (http://www.idsociety.org/Index.aspx) and CDC (http://www.cdc.gov/tb/publications/guidelines/ treatment.htm).

Representatives from the American Academy of Pediatrics, Canadian Thoracic Society, International Union Against Tuberculosis and Lung Disease, and World Health Organization also participated in the development of these guidelines, which update American Thoracic Society/CDC/IDSA guidelines published in 2003 (2). The guidelines have been endorsed by the European Respiratory Society and the U.S. National Tuberculosis Controllers Association.

The guidelines provide recommendations for the clinical and public health management of active tuberculosis in settings in which mycobacterial cultures, drug susceptibility testing, and radiographic studies are routinely available. For all recommendations, literature reviews were performed, followed by assessment of the quality of evidence, using the Grading of Recommendations, Assessment, Development and Evaluation methodology (3).

Given the public health implications of prompt diagnosis and effective management of tuberculosis, empiric multidrug treatment should be initiated in almost all situations in which active tuberculosis is suspected. Clinicians and public health practitioners who care for persons with active tuberculosis should see the full-text online version of the document, which provides detailed discussion of the management of drugsusceptible tuberculosis and recommendations for practice.

\section{References}

1. Nahid P, Dorman SE, Alipanah N, et al. Official American Thoracic Society/Centers for Disease Control and Prevention/Infectious Diseases Society of America Clinical Practice Guidelines: treatment of drugsusceptible tuberculosis. Clin Infect Dis 2016. Epub August 10, 2016. http://dx.doi.org/10.1093/cid/ciw376

2. American Thoracic Society; CDC; Infectious Diseases Society of America. Treatment of tuberculosis. MMWR Recomm Rep 2003;52(No. RR-11).

3. Guyatt GH, Oxman AD, Vist GE, et al.; GRADE Working Group. GRADE: an emerging consensus on rating quality of evidence and strength of recommendations. BMJ 2008;336:924-6. http://dx.doi.org/10.1136/ bmj.39489.470347.AD 